Thorax, 1978, ‘33, 662-663

\title{
Recurrent symptomatic pulmonary embolism caused by an infected Pudenz cerebrospinal fluid shunt device
}

\author{
R T N GIBNEY, F DONOVAN, AND M X FITZGERALD
}

From the Department of Respiratory Medicine and Neurosurgery, St Vincent's Hospital, Dublin, Eire

Subclinical recurrent pulmonary embolism from the atrial catheter tip is a recognised complication of ventriculoatrial shunting for hydrocephalus. Such recurrent pulmonary embolism is generally asymptomatic but may present with the insidious development of cor pulmonale (Talner et al, 1961; Friedman et al, 1964), or, more rarely, sudden death from massive embolisation. Classical symptomatic pulmonary infarction appears to be exceptional, with no case reported over the past 18 years. We report a case of severe symptomatic pulmonary infarction caused by septic emboli from an infected ventriculoatrial shunt (Pudenz valve).

\section{Case report}

A 25-year-old man was admitted for reinsertion of a Pudenz valve to relieve hydrocephalus, caused by a cerebellar tumour. In the 48 hours postoperatively he became dyspnoeic, febrile, and twice complained of right-sided pleuritic chest pain. Chest radiograph showed subsegmental bibasal atelectasis. The pain and pyrexia subsided on co-trimoxazole treatment. On the fourth postoperative day he again developed pleuritic pain, this time on the left side. Chest film was unchanged, but a lung scan showed small perfusion defects in the lingula and right upper lobe. In the absence of corresponding radiographic infiltrates pulmonary embolism was diagnosed, and the patient was treated with Heparin. Despite anticoagulation, a chest film 10 days later showed diminished vascular markings in the lower half of the right lung, and a scan showed a new massive defect with absent perfusion of the lower twothirds of the right lung (see figure). Since no other source of embolism, such as venous thrombosis, was apparent the episodes were attributed to emboli from the tip of the ventriculoatrial shunt. On removal, the atrial catheter tip was found to be occluded by a mass of organised thrombus. Culture of the catheter tip and associated thrombus isolated Staphylococcus epidermidis. After removal of the shunt the patient recovered, and his chest film and lung scan returned to normal.

\section{Discussion}

One necropsy study (Friedman et al, 1964) on patients with ventriculoatrial CSF shunts has shown evidence of old or recent pulmonary embolism in $58 \%$ as compared to $30 \%$ in a control group of unshunted hydrocephalics. Cor pulmonale of insidious onset caused by recurrent bland or septic pulmonary emboli from the atrial catheter tip is an uncommon complication of ventriculoatrial shunts. Sperling et al (1964) found clinically apparent cor pulmonale in two out of 30 patients with ventriculoatrial shunts. Massive pulmonary embolism complicating ventriculoatrial shunts is extremely rare, but may result in sudden death. Classical symptoms of pulmonary infarction, as occurred in this case, are exceptional, however, possibly because most shunts are performed in infants.

Talner et al (1961) stressed the importance of shunt infection in the production of pulmonary embolisation. The atrial catheter tip acts as a nidus for infection and subsequent infected thrombus. A recent review reported a shunt infection rate of $22 \%$. As in this case, the skin contaminant Staph epidermidis, acquired intraoperatively, is the most common pathogen, implicated in $60 \%$ of cases. Other organisms isolated include Staph aureus, Escherichia coli, pseudomonas, Proteus vulgaris, and Diphtheroid spp (Schoenbaum et al, 1975).

Antibiotics alone are usually inadequate, as in this case, where pulmonary embolism continued despite treatment. Complete removal of the shunt is required to eliminate reliably the source of infection and embolism (Schoenbaum et al, 1975).

Various screening techniques to detect embolism in the shunted patient have been suggested. Physical examination and electrocardiography, however, show abnormalities only when pulmonary hypertension is advanced. Lung scanning, on the other hand, has the advantage over pulmonary angiography of being safe and easily repeated, and has proved valuable in detecting embolism in shunted patients (Brisman et al, 1970). 




Figure Perfusion lung scan TC 99m MAA anterior view 3 August 1976. Large perfusion defect in lower two-thirds of right lung.

In this present case the evolution and resolution of pulmonary infarction was monitored by lungscanning. We think this technique should be used more often in the surveillance of patients with ventriculoatrial shunts.

\section{References}

Brisman, R, Stein, B M, and Johnson, P M (1970). Lung scan and shunted childhood hydrocephalus. Developmental Medicine and Child Neurology, 12, Supplement 22, 18-23.

Friedman, S, Zita-Gozum, C, and Chatten, J (1964). Pulmonary vascular changes complicating ventriculovascular shunting for hydrocephalus. Journal of Pediatrics, 64, 305-314.
Schoenbaum, S C, Gardner, P, and Shillito, J (1975). Infections of cerebrospinal fluid shunts: epidemiology, clinical manifestations and therapy. Journal of Infectious Diseases, 131, 543-552.

Sperling, D R, Patrick, J R, Anderson, F M, and Fyler, D C (1964). Cor pulmonale secondary to ventriculoauriculostomy. American Journal of Diseases of Children, 107, 308-315.

Talner, N S, Liu, H Y, Oberman, H A, and Schmidt, R W (1961). Thromboembolism complicating Holter valve shunt. American Journal of Diseases of Children, 101, 602-609.

Requests for reprints to: Professor M X FitzGerald, Department of Respiratory Medicine, St Vincent's Hospital, Dublin. 